\title{
Micro-Encapsulation of Probiotic Metabolites and Magnetic Nanoparticles Inside Liposomes using Microfluidic Devices
}

Luis M. Montes-de-Oca ${ }^{1}$, Fabiola Cuellar ${ }^{2}$, Maricela Rodriguez-Nieto' ${ }^{1}$, R. Rodríguez-López ${ }^{3}$, Said Aranda Espinoza ${ }^{3}$, Gabriel Espinosa ${ }^{1}$, Porfiria Barrón-González ${ }^{2}$, and Jorge Luis Menchaca ${ }^{4}$.

1. Instituto de Física y Matemáticas, Universidad Michoacana de San Nicolás de Hidalgo, Morelia, Michoacán, México.

2. Facultad de Ciencias Biológicas, Universidad Autónoma de Nuevo León, San Nicolás de los Garza, Nuevo León, México.

${ }^{3 .}$ Instituto de Física Manuel Sandoval Vallarta, Universidad Autónoma de San Luis Potosí Álvaro Obregón 64, 78000 San Luis Potosí, SLP, México.

4. Facultad de Ciencias Físico-Matemáticas, Universidad Autónoma de Nuevo León, San Nicolás de los Garza, Nuevo León, México.

Lipid vesicles are spherical structures constituted by a semipermeable membrane, which is formed by a lipid bilayer. They are regarded as a biomimetic technology due to their similarity with the plasmatic membrane of the cell. This similarity confers them the special characteristic to interact with living cells, through the controlled delivery of molecules to specific cells, being even possible the delivery of agents inside cells. In recent years, microfluidic technique using coaxial devices had shown to be efficient in the formation of monodisperse lipid vesicles from water-in-oil-in-water double emulsions with ultrathin oil shells used as templates[1]. In this work, some applications of these techniques are shown; the encapsulation of extracellular probiotic metabolites from the Lactobacillus plantarum strain, and the encapsulation of hydrophilic superparamagnetic nanoparticles. Administration of extracellular probiotic metabolites is presented as an alternative to the administration of live probiotic bacteria to overcome the intrinsic problems of these procedures, as the dependence of the host conditions on the growth and lifetime of probiotics[2]. Moreover, magnetic liposomes can be used as clinical contrast agents in magnetic resonance imaging (MRI), magnetic hyperthermia and drug delivery systems[3].

Lactobacillus plantarum (229, LP) strain was obtained from the American Type Culture Collection, Manassas, VA (ATCC, 1994). MRS-broth (Sigma) was used to grow probiotics for metabolites production. It was cultured by inoculating $1 \%$ of probiotics and incubated at $37^{\circ} \mathrm{C}$ for $24 \mathrm{~h}$. Probiotic metabolites were obtained from harvested probiotics, and centrifuged at $2500 \mathrm{rpm}$ for $10 \mathrm{~min}$. The supernatant was removed and sterilized with $0.22 \mu \mathrm{m}$ pore size Millipore filters. The obtained probiotic metabolites were frozen at $-40^{\circ} \mathrm{C}$ and lyophilized. Water-in-oil-in-water double emulsions droplets with ultrathin oil shells were obtained using glass tapered microfluidic devices with a procedure similar to that reported in [1]. The inner phase was an aqueous solution: for the encapsulation of nanoparticles, solutions of $8 \mathrm{wt} \%$ poly(ethylene glycol) (PEG) and $2 \mathrm{wt} \%$ poly(vinyl alcohol) (PVA) containing nanoparticles at different concentrations were used; for probiotic metabolites, the culture media of the probiotics was the same aqueous phase used in the microfluidic devices. The middle face consisted of lipid dissolutions in organic solvents: for the encapsulation of probiotic metabolites, a lipid mixture of $35 \mathrm{~mol} \%$ 1,2-dioleylsn-glycero-3-phosphocholine (DOPC), 35 mol\% 1,2-dipalmitoyl-sn-glycero-3-phosphocholine (DPPC) and $30 \mathrm{~mol} \%$ cholesterol was used, while superparamagnetic liposomes were prepared with pure DPPC. In both cases, the lipid molecules were dissolved in a mixture of $36 \%$ chloroform and $64 \%$ hexane $\mathrm{v} / \mathrm{v}$; at a total lipid concentration of $5 \mathrm{mg} / \mathrm{mL}$. The outer phase was an aqueous solution of $10 \%$ PVA in all cases. The flow ratios used for the inner, middle and outer phases were $\mathrm{Q}_{\text {inn }}=8.33 \mu \mathrm{L} / \mathrm{min}, \mathrm{Q}_{\text {middle }}=8.33$ 
$\mu \mathrm{L} / \mathrm{min}$ and $\mathrm{Q}_{\text {out }}=50 \mu \mathrm{L} / \mathrm{min}$ for nanoparticles and $\mathrm{Q}_{\text {inn }}=8.33 \mu \mathrm{L} / \mathrm{min}, \mathrm{Q}_{\text {middle }}=8.33 \mu \mathrm{L} / \mathrm{min}$ and $\mathrm{Q}_{\text {out }}=$ $140 \mu \mathrm{L} / \mathrm{min}$ for metabolites. This difference arises from the properties of the culture medium where the metabolites were dispersed, which contains organic surfactant compounds and possess a viscosity lower than the PEG-PVA solution. In all cases, the samples were collected in sucrose water solutions to match the osmotic pressure of the media inside the vesicles.

Figure 1 shows the microfluidic device used to form the double emulsion templates and their formation stage. The high control over the flow ratios allow the formation of highly monodisperse double emulsion drops, which become in monodisperse unilamellar lipid vesicles, as it is shown in figures $2 \mathrm{a}$ and $2 \mathrm{~b}$. Figure 2c shows a lipid vesicle containing probiotics metabolites, dyed with fluorescein sodium salt (FSS). Our results showed some of the possibilities that can be exploited by the microfluidic technique. We showed that the encapsulation of an aqueous solution with low viscosity as the growth media of the probiotics is achievable by increasing the flow ratio of the outer phase in the microfluidic devices. In the case of the paramagnetic nanoparticles, we observed that the number and stability of the vesicles decreased as the concentration of nanoparticles increases.

References:

[1] L.R. Arriaga et al, Small. 10 (2014), p. 950.

[2] M. José et al, Innov. Food Emerg. Technol. 27 (2015), p. 15.

[3] L.A. Tai et al, Nanotechnology. 20 (2009).

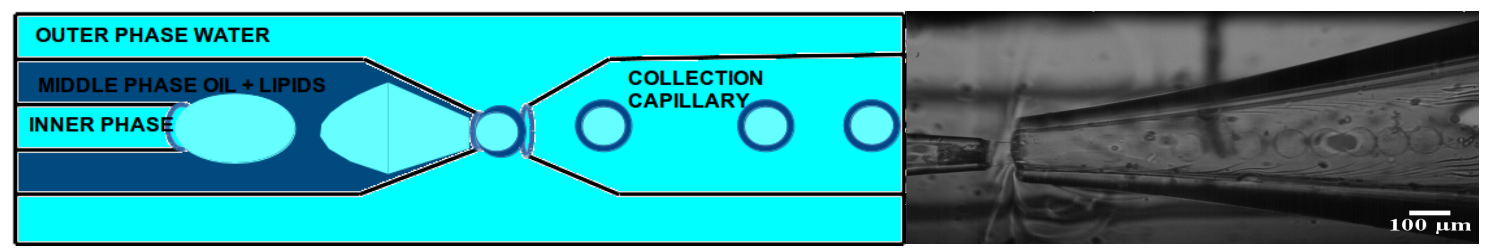

Figure 1. Left: Schematic diagram of the microfluidic device. (b) Right: Optical microscopy image of the production of the double emulsion templates to form vesicles in the microfluidic device.

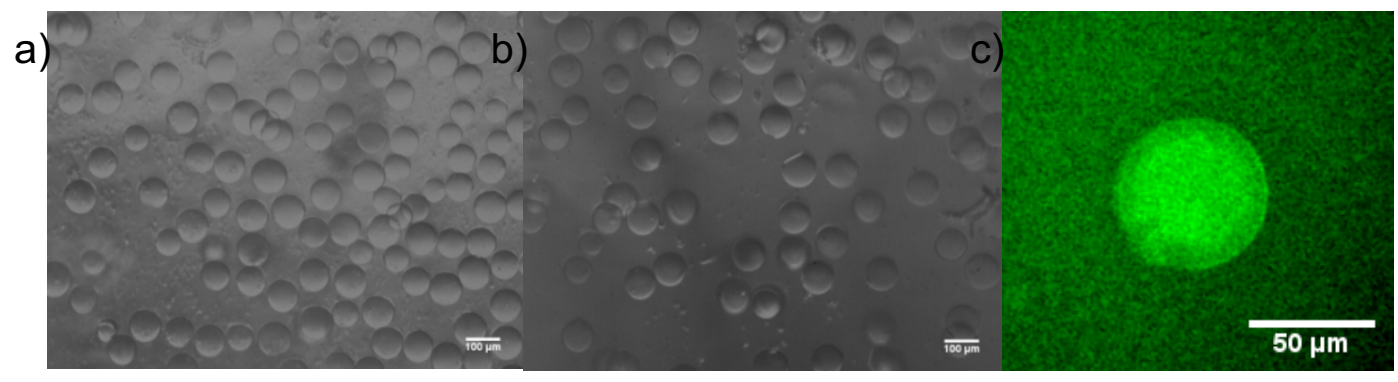

Figure 2. Optical micrographs of vesicles with solution of nanoparticles, encapsulated at (a) $1 \% \mathrm{v} / \mathrm{v}$, which remained stable during 11 days, and (b) 5\% v/v, which remained stable during 5 days. (c) Fluorescence micrograph using a LED excitation source of $365 \mathrm{~nm}$ wavelength, it shows a lipid vesicle with extracellular probiotic metabolites dyed with FSS and encapsulated into the core, observed 25 days after formation. 